\title{
Cultivares de alfafa em área de influência da Mata Atlântica no Estado de Minas Gerais(1)
}

\author{
Milton de Andrade Botrel(2), Reinaldo de Paula Ferreira(2), Maurílio José Alvim ${ }^{(2)}$ e Deise Ferreira Xavier ${ }^{(3)}$
}

Resumo - Este trabalho teve como objetivo avaliar o potencial forrageiro de cultivares de alfafa (Medicago sativa L.) em área de influência da Mata Atlântica no Estado de Minas Gerais. Foram avaliadas 30 cultivares de alfafa em delineamento de blocos ao acaso com três repetições. As sementes foram infectadas com a estirpe de Rhizobium melilotii BR 7407. No período das chuvas e da seca houve diferenças significativas entre as cultivares quanto ao potencial de produção de forragem, relação folha/caule e tolerância a pragas e doenças. Quanto ao teor de proteína bruta, houve diferenças significativas entre as cultivares, somente no período das chuvas. As cultivares que se destacaram na maioria dos parâmetros avaliados foram a Crioula, P30, Monarca e Flórida 77. As maiores produções de forragem nas estações das chuvas e da seca foram obtidas pela cultivar Crioula, constituindo, assim, boa opção para o cultivo da alfafa na Zona da Mata de Minas Gerais.

Termos para indexação: forragem verde, proteína bruta, valor nutritivo, fatores de rendimento.

Alfalfa cultivars under areas of influence of the Atlantic Forest in the Minas Gerais State, Brazil

Abstract - The aim of this work was to evaluate the yields of alfalfa (Medicago sativa L.) cultivars for the region under influence of the Atlantic Forest in Minas Gerais, Brazil. Thirty cultivars were evaluated in a randomized block design with three replications. Before planting, seeds were inoculated with Rhizobium melilotii BR 7407. During the dry and rainy season, significant differences were observed among cultivars for forage production, leaf:stem ratio, and insect and disease resistance. Regarding the crude protein content in the forage, significant differences were observed among cultivars only during the rainy season. Crioula, P30, Monarca and Florida 77 cultivars had the best performance for the majority of evaluated parameters. High forage production during rainy and dry seasons was obtained with Crioula cultivar, which should be recommended as the cultivar to be grown for forage crop in the region of this study.

Index terms: green feed, crude protein, nutritive value, yield factors.

\section{Introdução}

Devido ao seu alto rendimento e à qualidade de sua forragem, a alfafa é uma das forrageiras mais utilizadas em todo o mundo. Em razão disso, é um dos volumosos mais indicados para rebanhos leiteiros especializados (Bolland, 1994; Comerón, 1994; Vilela, 1994; Castillo \& Gallardo, 1995).

(1) Aceito para publicação em 18 de maio de 2001.

(2) Embrapa-Centro Nacional de Pesquisa de Gado de Leite (CNPGL), Rua Eugênio do Nascimento, 610, CEP 36038-330 Juiz de Fora, MG. Bolsista do CNPq. E-mail: mbotre1@cnpgl.embrapa.br, ferreira@cnpgl.embrapa.br, alvim@cnpgl.embrapa.br

(3)Embrapa-CNPGL. E-mail: dfxavier@cnpgl.embrapa.br
As pesquisas com a alfafa em regiões tropicais, principalmente no Brasil, são recentes (Rassini \& Freitas, 1995; Botrel \& Alvim, 1997; Evangelista et al., 1998; Rassini \& Freitas, 1998; Ruggieri et al., 1998; Viana et al., 1998), e sempre indicam essa leguminosa como volumoso de alta produtividade e valor nutritivo. Entretanto, o aproveitamento do potencial máximo dessa leguminosa como forrageira poderá ser comprometido pela inexistência de conhecimentos sobre a adaptação, produção e utilização dessa leguminosa, nos diversos ambientes tropicais em que serão utilizadas.

Na Região Sudeste estão concentradas as maiores bacias leiteiras do País (Zoccal, 1994). Nessa região tem ocorrido uma intensificação dos sistemas de produção de leite, e a alfafa poderá contri- 
buir como alimento volumoso para a alimentação do rebanho leiteiro especializado, mantido nesses sistemas. A Embrapa-Centro Nacional de Pesquisa de Gado de Leite coordena uma rede nacional de ensaios com alfafa, com o objetivo de identificar cultivares adaptadas às diferentes condições de solo e clima das principais bacias leiteiras do País.

O objetivo deste trabalho foi avaliar o potencial forrageiro de cultivares de alfafa na Região da Zona da Mata de Minas Gerais, em área de influência da Mata Atlântica.

\section{Material e Métodos}

O experimento foi conduzido durante o período de dezembro de 1994 a janeiro de 1997, no campo experimental da Embrapa-Centro Nacional de Pesquisa de Gado de Leite, localizado no Estado de Minas Gerais, na latitude $21^{\circ} 33^{\prime} \mathrm{S}$ e longitude $43^{\circ} 6^{\prime} \mathrm{W}$ e altitude de $426 \mathrm{~m}$. O clima da região é do tipo Cwa, mesotérmico, apresentando verão quente e chuvoso e inverno frio e seco. A precipitação anual está em torno de $1.500 \mathrm{~mm}$, e a temperatura média dos meses mais frios é de $18^{\circ} \mathrm{C}$, e dos mais quentes, $22^{\circ} \mathrm{C}$. O experimento foi conduzido em Latossolo Vermelho-Amarelo, de relevo acidentado. As amostras de solo coletadas nas profundidades de $0-20$ e $20-40 \mathrm{~cm}$ revelaram, respectivamente, os seguintes valores: $\mathrm{pH}$ em água 5,39 e 5,$00 ; 0,30$ e $0,24 \mathrm{cmol}_{\mathrm{c}} \mathrm{dm}^{-3} \mathrm{de}^{\mathrm{Al}^{3+}} ; 2,74 \mathrm{e}$ $1,55 \mathrm{cmol}_{\mathrm{c}} \mathrm{dm}^{-3} \mathrm{de} \mathrm{Ca}^{2+}+\mathrm{Mg}^{2+} ; 0,10$ e $0,05 \mathrm{cmol}_{\mathrm{c}} \mathrm{dm}^{-3} \mathrm{de}$ $\mathrm{K}^{+}$e 3,68 e $1,16 \mathrm{mg} \mathrm{dm}^{-3}$ de $\mathrm{P}$.

A quantidade de calcário dolomítico aplicada (2,5 t/ha) visou atingir o nível de $80 \%$ de saturação por bases. O calcário foi aplicado 60 dias antes da semeadura e incorporado ao solo por meio de uma aração a $40 \mathrm{~cm}$ de profundidade, seguida de duas gradagens. Por ocasião da semeadura, aplicaram-se $40 \mathrm{~kg} /$ ha de $\mathrm{P}_{2} \mathrm{O}_{5}$ (superfosfato simples), que foram incorporados ao solo por meio de gradagem superficial. Nessa mesma ocasião (15/12/94), foram aplicados e incorporados, nos sulcos de semeadura, $50 \mathrm{~kg} / \mathrm{ha} \mathrm{de} \mathrm{P}_{2} \mathrm{O}_{5}$ (superfosfato simples), $87 \mathrm{~kg} /$ ha de $\mathrm{K}_{2} \mathrm{O}$ (cloreto de potássio) e $40 \mathrm{~kg} / \mathrm{ha}$ de FTE-BR 16 Em seguida, foi realizada a semeadura, a uma profundidade de $2 \mathrm{~cm}$ e a uma taxa de $20 \mathrm{~kg} / \mathrm{ha}$, de sementes puras viáveis. As sementes foram infectadas com a estirpe de Rhizobium melilotii BR 7407, proveniente da EmbrapaCentro Nacional de Pesquisa de Agrobiologia, e peletizadas com calcário dolomítico. A adubação de manutenção consistiu na aplicação anual de $120 \mathrm{~kg} / \mathrm{ha}$ de $\mathrm{P}_{2} \mathrm{O}_{5}$ (superfosfato simples) e $360 \mathrm{~kg} / \mathrm{ha}$ de $\mathrm{K}_{2} \mathrm{O}$ (cloreto de potássio)
O delineamento experimental utilizado foi o de blocos completos ao acaso, com três repetições. As parcelas foram constituídas de cinco fileiras de $5 \mathrm{~m}$ de comprimento, com espaçamento de $30 \mathrm{~cm}(5,0 \times 5,0 \times 0,30 \mathrm{~m})$. Considerou-se como bordadura uma fileira de cada lado, e $0,50 \mathrm{~m}$ de cada extremidade da parcela. Os tratamentos consistiram de 30 cultivares de alfafa.

As cultivares foram avaliadas no período de 21/2/95 a 22/1/97, quanto às seguintes características: produção de matéria seca, relação folha/caule, teor de proteína bruta nas folhas, no caule e na planta inteira, e tolerância a pragas e doenças. Os cortes para estimativa da produção da forragem foram feitos a uma altura de $5 \mathrm{~cm}$ do solo e na área útil de cada parcela, sempre que mais de $50 \%$ das cultivares atingiam $10 \%$ de floração (aparecimento das primeiras flores). Esse manejo permitiu obter, em média, seis cortes durante a estação das chuvas, e três na da seca. $\mathrm{Na}$ forragem verde colhida para determinação da produção de forragem, foram retiradas, aleatoriamente, 50 hastes de cada parcela, e separadas manualmente as folhas dos caules, para posterior determinação da relação folha/caule e do teor de proteína bruta de cada componente, conforme Association of Official Analytical Chemists (1970).

A susceptibilidade a pragas e doenças foi avaliada conforme Hijano (1994), através da estimativa visual em cada parcela da porcentagem de plantas resistentes a doenças ou a pragas, e as plantas foram classificadas como: Susceptível ( $0-5 \%$ de plantas resistentes); Baixa Resistência (6-14\% de plantas resistentes); Moderadamente Resistentes $(15-50 \%$ de plantas resistentes); e Resistentes $(>50 \%$ de plantas resistentes)

A irrigação foi feita por aspersão, principalmente no período da seca, tomando-se como base a leitura de tensiômetro de cápsula porosa e em coluna de mercúrio (calibrado para $60 \%$ de umidade do solo), resultando, em média, uma freqüência semanal de irrigação que correspondeu a uma lâmina d'água de $30 \mathrm{~mm}$.

Os resultados foram submetidos à análise de variância através do pacote estatístico SAEG, e as médias, comparadas pelo teste de agrupamentos de Scott-Knott (Euclydes, 1988).

\section{Resultados e Discussão}

Houve diferenças significativas $(\mathrm{P}<0,05)$ entre as cultivares quanto ao potencial de produção de forragem (Tabela 1).

A maior produção anual de forragem $(13.008 \mathrm{~kg} / \mathrm{ha}$ de matéria seca) foi obtida pela cultivar Crioula, sendo $34,4 \%$ superior ao rendimento médio das demais 
cultivares $(8.534 \mathrm{~kg} / \mathrm{ha})$ em avaliação, o que mostra a sua boa adaptação às condições tropicais (Tabela 1). Outras cultivares que também se destacaram com relação à produção anual de forragem foram a Monarca, P30 e Flórida 77, apresentando rendimentos semelhantes, porém inferiores ao do padrão (cultivar Crioula). Evangelista et al. (1998) e Viana et al. (1998) também constataram o alto potencial forrageiro das cultivares Crioula e P30 no Estado de Minas Gerais.

$\mathrm{Na}$ época da seca, a produção média de forragem, de todas as cultivares, foi cerca de $30 \%$ do rendi- mento médio anual, o que indica a alfafa como recurso forrageiro alternativo para o rebanho leiteiro durante o período da seca, quando o crescimento das espécies tropicais, mesmo em condições de irrigação, é baixo (Botrel et al., 1991). Nesse período, a cultivar Crioula também se destacou em relação às demais quanto ao rendimento forrageiro. Esse mesmo comportamento repetiu-se na época das chuvas, seguida das cultivares Monarca, P30 e Flórida 77.

A concentração de proteína bruta nas folhas variou de $36,2 \%$ (cultivar Flórida 77 ) a $29,9 \%$ (cultivar BR1), com um valor médio de 32,9\% (Ta-

Tabela 1. Produção de matéria seca, teor de proteína bruta na folha (F), no caule (C) e na planta inteira (PI) de cultivares de alfafa, avaliadas durante o período da seca e das chuvas ${ }^{(1)}$.

\begin{tabular}{|c|c|c|c|c|c|c|c|c|c|}
\hline \multirow[t]{3}{*}{ Cultivar } & \multicolumn{3}{|c|}{ Matéria seca (kg/ha) } & \multicolumn{6}{|c|}{ Proteína bruta (\%) } \\
\hline & \multirow[t]{2}{*}{ Anual } & \multirow[t]{2}{*}{ Seca } & \multirow[t]{2}{*}{ Chuva } & \multicolumn{3}{|c|}{ Seca } & \multicolumn{3}{|c|}{ Chuva } \\
\hline & & & & $\mathrm{F}$ & $\mathrm{C}$ & PI & $\mathrm{F}$ & $\mathrm{C}$ & PI \\
\hline Crioula & $13.008 \mathrm{~A}$ & $4.048 \mathrm{~A}$ & $8.960 \mathrm{~A}$ & $32,6 \mathrm{~A}$ & $19,7 \mathrm{~A}$ & $27,0 \mathrm{~A}$ & $31,8 \mathrm{~B}$ & $17,7 \mathrm{~B}$ & $24,9 \mathrm{~B}$ \\
\hline Monarca & $11.916 \mathrm{~B}$ & $3.718 \mathrm{~B}$ & $8.198 \mathrm{~B}$ & $36,9 \mathrm{~A}$ & $22,0 \mathrm{~A}$ & $30,5 \mathrm{~A}$ & $35,4 \mathrm{~A}$ & $23,5 \mathrm{~A}$ & $29,8 \mathrm{~A}$ \\
\hline P30 & $11.841 \mathrm{~B}$ & $3.583 \mathrm{~B}$ & $8.258 \mathrm{~B}$ & $33,0 \mathrm{~A}$ & $19,7 \mathrm{~A}$ & $26,7 \mathrm{~A}$ & $35,6 \mathrm{~A}$ & $21,1 \mathrm{~A}$ & $28,5 \mathrm{~A}$ \\
\hline Flórida 77 & $11.813 \mathrm{~B}$ & $3.698 \mathrm{~B}$ & $8.115 \mathrm{~B}$ & $37,4 \mathrm{~A}$ & $22,2 \mathrm{~A}$ & $30,8 \mathrm{~A}$ & $36,2 \mathrm{~A}$ & $23,0 \mathrm{~A}$ & $29,7 \mathrm{~A}$ \\
\hline Valley Plus & $11.400 \mathrm{C}$ & $3.668 \mathrm{~B}$ & $7.732 \mathrm{C}$ & $35,7 \mathrm{~A}$ & $22,0 \mathrm{~A}$ & $29,8 \mathrm{~A}$ & $35,0 \mathrm{~A}$ & $21,1 \mathrm{~A}$ & $27,7 \mathrm{~A}$ \\
\hline SW $9210 \mathrm{~A}$ & $11.050 \mathrm{C}$ & $3.507 \mathrm{~B}$ & $7.543 \mathrm{C}$ & $34,1 \mathrm{~A}$ & $20,0 \mathrm{~A}$ & $27,7 \mathrm{~A}$ & $33,4 \mathrm{~B}$ & $18,0 \mathrm{~B}$ & $25,7 \mathrm{~B}$ \\
\hline P 5715 & $10.816 \mathrm{D}$ & $3.216 \mathrm{C}$ & $7.600 \mathrm{C}$ & $32,9 \mathrm{~A}$ & $20,4 \mathrm{~A}$ & $26,7 \mathrm{~A}$ & $32,4 \mathrm{~B}$ & $19,2 \mathrm{~B}$ & $25,2 \mathrm{~B}$ \\
\hline SW 8112 A & 10.471D & $3.274 \mathrm{C}$ & 7.197D & $34,1 \mathrm{~A}$ & $18,9 \mathrm{~A}$ & $27,5 \mathrm{~A}$ & $32,4 \mathrm{~B}$ & $20,1 \mathrm{~B}$ & $25,8 \mathrm{~B}$ \\
\hline Rio & $10.246 \mathrm{D}$ & $3.132 \mathrm{C}$ & $7.114 \mathrm{D}$ & $34,8 \mathrm{~A}$ & $22,2 \mathrm{~A}$ & $29,3 \mathrm{~A}$ & $33,0 \mathrm{~B}$ & $22,0 \mathrm{~A}$ & $27,6 \mathrm{~A}$ \\
\hline Maricopa & $10.232 \mathrm{D}$ & $3.164 \mathrm{C}$ & $7.068 \mathrm{D}$ & $33,6 \mathrm{~A}$ & $19,2 \mathrm{~A}$ & $27,5 \mathrm{~A}$ & $32,6 \mathrm{~B}$ & $19,3 \mathrm{~B}$ & $25,5 \mathrm{~B}$ \\
\hline El Grand & $7.148 \mathrm{D}$ & $3.056 \mathrm{C}$ & 7.089D & $37,4 \mathrm{~A}$ & $20,7 \mathrm{~A}$ & $29,6 \mathrm{~A}$ & $36,0 \mathrm{~A}$ & $21,2 \mathrm{~A}$ & $28,9 \mathrm{~A}$ \\
\hline P 5929 & $10.023 \mathrm{D}$ & 2.914D & 7.109D & $34,9 \mathrm{~A}$ & $22,6 \mathrm{~A}$ & $29,0 \mathrm{~A}$ & $33,6 \mathrm{~A}$ & $21,8 \mathrm{~A}$ & $27,5 \mathrm{~A}$ \\
\hline WL 516 & 9.868D & $3.377 \mathrm{C}$ & $6.491 \mathrm{E}$ & $34,6 \mathrm{~A}$ & $22,7 \mathrm{~A}$ & $29,1 \mathrm{~A}$ & $32,4 \mathrm{~B}$ & $23,1 \mathrm{~A}$ & $27,6 \mathrm{~A}$ \\
\hline Falcom & $9.226 \mathrm{E}$ & $3.137 \mathrm{C}$ & $6.089 \mathrm{~F}$ & $36,7 \mathrm{~A}$ & $23,6 \mathrm{~A}$ & $30,8 \mathrm{~A}$ & $33,1 \mathrm{~B}$ & $23,6 \mathrm{~A}$ & $28,3 \mathrm{~A}$ \\
\hline MH 04 & $8.775 \mathrm{~F}$ & $2.748 \mathrm{D}$ & $6.027 \mathrm{~F}$ & $35,0 \mathrm{~A}$ & $20,7 \mathrm{~A}$ & $28,2 \mathrm{~A}$ & $34,4 \mathrm{~A}$ & $21,6 \mathrm{~A}$ & $27,0 \mathrm{~A}$ \\
\hline Alto & $8.412 \mathrm{~F}$ & $2.642 \mathrm{E}$ & $5.770 \mathrm{~F}$ & $33,6 \mathrm{~A}$ & $20,5 \mathrm{~A}$ & $27,1 \mathrm{~A}$ & $33,3 \mathrm{~B}$ & $21,2 \mathrm{~A}$ & $26,9 \mathrm{~A}$ \\
\hline MH 15 & $7.955 \mathrm{G}$ & 2.903D & $5.052 \mathrm{G}$ & $35,3 \mathrm{~A}$ & $22,2 \mathrm{~A}$ & $29,7 \mathrm{~A}$ & $30,6 \mathrm{~B}$ & $18,5 \mathrm{~B}$ & $24,0 \mathrm{~B}$ \\
\hline BR 2 & $7.673 \mathrm{H}$ & $2.504 \mathrm{E}$ & $5.169 \mathrm{G}$ & $36,2 \mathrm{~A}$ & $21,0 \mathrm{~A}$ & $29,0 \mathrm{~A}$ & $33,8 \mathrm{~A}$ & $26,0 \mathrm{~A}$ & $27,1 \mathrm{~A}$ \\
\hline SW 8210 & $7.394 \mathrm{H}$ & $2.250 \mathrm{~F}$ & $5.144 \mathrm{G}$ & $34,2 \mathrm{~A}$ & $19,6 \mathrm{~A}$ & $27,9 \mathrm{~A}$ & $32,4 \mathrm{~B}$ & $18,0 \mathrm{~B}$ & $25,3 \mathrm{~B}$ \\
\hline Costera & $7.163 \mathrm{H}$ & $2.333 \mathrm{~F}$ & $4.830 \mathrm{G}$ & $36,1 \mathrm{~A}$ & $23,0 \mathrm{~A}$ & $30,5 \mathrm{~A}$ & $31,6 \mathrm{~B}$ & $24,0 \mathrm{~A}$ & $27,3 \mathrm{~A}$ \\
\hline Alfa 200 & $7.140 \mathrm{H}$ & $2.252 \mathrm{~F}$ & $4.888 \mathrm{G}$ & $34,7 \mathrm{~A}$ & $21,2 \mathrm{~A}$ & $28,5 \mathrm{~A}$ & $32,7 \mathrm{~B}$ & $21,8 \mathrm{~A}$ & $26,7 \mathrm{~B}$ \\
\hline P 5888 & $7.006 \mathrm{H}$ & $2.292 \mathrm{~F}$ & $4.714 \mathrm{G}$ & $34,6 \mathrm{~A}$ & $21,0 \mathrm{~A}$ & $28,1 \mathrm{~A}$ & $31,7 \mathrm{~B}$ & $21,1 \mathrm{~A}$ & $25,8 \mathrm{~B}$ \\
\hline ICI 990 & $6.876 \mathrm{H}$ & $2.006 \mathrm{G}$ & $4.870 \mathrm{G}$ & $34,3 \mathrm{~A}$ & $20,9 \mathrm{~A}$ & $28,1 \mathrm{~A}$ & $31,5 \mathrm{~B}$ & $21,0 \mathrm{~A}$ & $25,6 \mathrm{~B}$ \\
\hline Sutter & $6.818 \mathrm{H}$ & $2.103 \mathrm{~F}$ & $4.715 \mathrm{G}$ & $36,2 \mathrm{~A}$ & $21,5 \mathrm{~A}$ & $29,9 \mathrm{~A}$ & $31,5 \mathrm{~B}$ & $22,0 \mathrm{~A}$ & $26,3 \mathrm{~B}$ \\
\hline BR 3 & $6.584 \mathrm{I}$ & $1.994 \mathrm{G}$ & $4.590 \mathrm{G}$ & $34,7 \mathrm{~A}$ & $22,8 \mathrm{~A}$ & $30,0 \mathrm{~A}$ & $33,0 \mathrm{~B}$ & $20,6 \mathrm{~A}$ & $26,4 \mathrm{~B}$ \\
\hline Araucana & $6.408 \mathrm{I}$ & $1.797 \mathrm{G}$ & $4.611 \mathrm{G}$ & $34,8 \mathrm{~A}$ & $22,5 \mathrm{~A}$ & $29,6 \mathrm{~A}$ & $34,0 \mathrm{~A}$ & $22,0 \mathrm{~A}$ & $27,6 \mathrm{~A}$ \\
\hline Esmeralda & $6.525 \mathrm{I}$ & $1.906 \mathrm{G}$ & $4.619 \mathrm{G}$ & $34,3 \mathrm{~A}$ & $21,2 \mathrm{~A}$ & $28,7 \mathrm{~A}$ & $33,0 \mathrm{~B}$ & $21,5 \mathrm{~A}$ & $26,6 \mathrm{~B}$ \\
\hline BR 4 & $5.688 \mathrm{~J}$ & $2.005 \mathrm{G}$ & $3.683 \mathrm{H}$ & $35,3 \mathrm{~A}$ & $20,5 \mathrm{~A}$ & $28,9 \mathrm{~A}$ & $30,9 \mathrm{~B}$ & $19,6 \mathrm{~B}$ & $24,8 \mathrm{~B}$ \\
\hline Semith 921 & $6.126 \mathrm{~K}$ & $1.660 \mathrm{H}$ & $5.465 \mathrm{H}$ & $31,2 \mathrm{~A}$ & $19,4 \mathrm{~A}$ & $25,9 \mathrm{~A}$ & $30,0 \mathrm{~B}$ & $20,3 \mathrm{~B}$ & $24,9 \mathrm{~B}$ \\
\hline BR 1 & $4.908 \mathrm{~K}$ & $1.425 \mathrm{H}$ & $3.483 \mathrm{H}$ & $33,3 \mathrm{~A}$ & $22,3 \mathrm{~A}$ & $29,0 \mathrm{~A}$ & $29,9 \mathrm{~B}$ & $22,2 \mathrm{~A}$ & $25,6 \mathrm{~B}$ \\
\hline Média & 8.684 & 2.610 & 6.106 & 34,8 & 21,2 & 28,7 & 32,9 & 21,1 & 26,7 \\
\hline
\end{tabular}

${ }^{(1)}$ Médias seguidas pela mesma letra na coluna não diferem entre si a 5\% de probabilidade pelo teste de Scott-Knott. 
bela 1). Os valores extremos do teor de proteína bruta no caule foram de $23,6 \%$ (cultivar Falcon) e 17,7\% (cultivar Crioula). O teor médio de proteína na planta inteira da cultivar Crioula foi de $24,9 \%$ e o valor médio de todas as cultivares de 26,7\%. Aproximadamente 30,70 e $46 \%$ das cultivares avaliadas foram superiores ao padrão (cultivar Crioula), quanto ao teor de proteína nas folhas, no caule e na planta inteira, respectivamente.

Durante o período da seca, as cultivares não diferiram quanto ao teor de proteína bruta nas folhas, caules e na planta inteira, cujos valores médios foram $34,8,21,2$ e $28,7 \%$, respectivamente.

O teor de proteína bruta nas folhas foi alto, em geral, sendo em média, $56 \%$ e $64 \%$ superiores aos valores observados no caule, respectivamente, durante o período das águas e da seca. Resultados semelhantes foram obtidos por Marten et al. (1988), que, como Botrel \& Alvim (1996), ressaltam a importância de se manter, através de manejo adequado, maior proporção de folhas na forragem colhida, obtendo-se, assim, um alimento de alto valor nutritivo, principalmente durante o período da seca, quando o valor nutritivo das espécies tropicais cai acentuadamente (Alvim et al., 1986; Botrel et al., 1991).

Houve tendência de o teor médio de proteína bruta da planta inteira ser maior durante a época da seca em relação à época das chuvas (Tabela 1). Este fato está provavelmente associado com a menor proporção de folhas na forragem colhida durante a estação das águas (Tabela 2), e também com as maiores produções observadas nesse período, causando uma diluição desse nutriente na matéria seca produzida.

A relação folha/caule variou de 1,58 (cultivar BR3) a 1,01 (cultivar P 5715) e de 1,12 (cultivar Monarca) a 0,75 (cultivar MH4), respectivamente no período da seca e das chuvas (Tabela 2).

$\mathrm{Na}$ estação das chuvas, a relação folha/caule da cultivar Crioula foi 1,05 , situando-se no grupo das cultivares que apresentaram maior proporção de folhas na forragem colhida. No período da seca, cerca de $6 \%$ e $46 \%$ das cultivares apresentaram, respectivamente, maior e menor proporção de folhas em relação ao padrão (cultivar Crioula). A relação folha/ caule de todas as cultivares foi sempre menor nas avaliações feitas durante o período das chuvas, ou seja, $42 \%$ do valor observado durante a estação da seca.
A incidência de doenças foi mais freqüente durante o período das chuvas (Tabela 2), provavelmente devido às condições climáticas predominantes nessa época do ano (temperatura e umidade elevadas), que são favoráveis ao desenvolvimento das principais doenças da alfafa (Melton et al., 1988). Assim, nas avaliações realizadas, respectivamente, durante o período da seca e das chuvas, 43 e $10 \%$ das cultivares situaram-se no grupo das resistentes a doenças. Segundo Marten et al. (1988), as doenças foliares causam senescência e queda das folhas, aumentando a proporção de caules, resultando, conseqüentemente, em menor concentração de proteína bruta na matéria seca da alfafa. De fato, houve tendência, principalmente durante o período das chuvas, de as cultivares mais susceptíveis a doenças apresentarem maior proporção de caule, com reflexos negativos

Tabela 2. Relação folha/caule (F/C), tolerância a doenças (TD) e pragas (TP) de cultivares de alfafa, avaliadas durante o período da seca e das chuva ${ }^{(1)}$

\begin{tabular}{|c|c|c|c|c|c|c|}
\hline \multirow[t]{2}{*}{ Cultivar } & \multicolumn{2}{|c|}{$\mathrm{F} / \mathrm{C}$} & \multicolumn{2}{|c|}{ TD } & \multicolumn{2}{|c|}{$\mathrm{TP}$} \\
\hline & Seca & Chuva & Seca & Chuva & Seca & Chuva \\
\hline BR 3 & $1,58 \mathrm{~A}$ & $0,88 \mathrm{C}$ & MR & $\mathrm{S}$ & MR & $\mathrm{R}$ \\
\hline BR 1 & $1,57 \mathrm{~A}$ & $0,78 \mathrm{D}$ & MR & $\mathrm{S}$ & MR & $\mathrm{R}$ \\
\hline Araucana & $1,39 \mathrm{~B}$ & $0,87 \mathrm{C}$ & $\mathrm{R}$ & $\mathrm{S}$ & $\mathrm{R}$ & $\mathrm{R}$ \\
\hline Sutter & $1,35 \mathrm{~B}$ & $0,82 \mathrm{D}$ & MR & BR & MR & $\mathrm{R}$ \\
\hline Crioula & $1,35 \mathrm{~B}$ & $1,05 \mathrm{~A}$ & $\mathrm{R}$ & $\mathrm{R}$ & $\mathrm{R}$ & $\mathrm{R}$ \\
\hline Monarca & $1,34 \mathrm{~B}$ & $1,12 \mathrm{~A}$ & $\mathrm{R}$ & MR & $\mathrm{R}$ & $\mathrm{R}$ \\
\hline Esmeralda & $1,34 \mathrm{~B}$ & $0,80 \mathrm{D}$ & MR & MR & MR & $\mathrm{R}$ \\
\hline Costera & $1,34 \mathrm{~B}$ & $0,77 \mathrm{D}$ & MR & MR & MR & $\mathrm{R}$ \\
\hline Maricopa & $1,33 \mathrm{~B}$ & $0,88 \mathrm{C}$ & BR & $\mathrm{S}$ & MR & $\mathrm{R}$ \\
\hline MH 15 & $1,33 \mathrm{~B}$ & $0,83 \mathrm{D}$ & $\mathrm{BR}$ & $\mathrm{S}$ & MR & MR \\
\hline SW 8210 & $1,32 \mathrm{~B}$ & $0,80 \mathrm{D}$ & MR & MR & MR & $\mathrm{R}$ \\
\hline Valley Plus & $1,32 \mathrm{~B}$ & $0,91 \mathrm{C}$ & $\mathrm{R}$ & MR & MR & $\mathrm{R}$ \\
\hline BR 4 & $1,31 \mathrm{~B}$ & $0,85 \mathrm{C}$ & BR & $\mathrm{S}$ & MR & $\mathrm{R}$ \\
\hline Rio & $1,31 \mathrm{~B}$ & $1,00 \mathrm{~B}$ & MR & BR & MR & MR \\
\hline Flórida 77 & $1,29 \mathrm{~B}$ & $1,02 \mathrm{~A}$ & MR & MR & $\mathrm{R}$ & $\mathrm{R}$ \\
\hline SW $8112 \mathrm{~A}$ & $1,29 \mathrm{~B}$ & $0,88 \mathrm{D}$ & $\mathrm{R}$ & BR & MR & $\mathrm{R}$ \\
\hline ICI 990 & $1,23 \mathrm{C}$ & $0,80 \mathrm{D}$ & BR & BR & BR & $\mathrm{R}$ \\
\hline Falcom & $1,23 \mathrm{C}$ & $0,99 \mathrm{~B}$ & MR & BR & MR & MR \\
\hline Alto & $1,22 \mathrm{C}$ & $0,88 \mathrm{C}$ & MR & $\mathrm{BR}$ & $\mathrm{R}$ & $\mathrm{R}$ \\
\hline SW $9210 \mathrm{~A}$ & $1,22 \mathrm{C}$ & $1,00 \mathrm{~B}$ & $\mathrm{R}$ & MR & MR & $\mathrm{R}$ \\
\hline Semit 921 & $1,22 \mathrm{C}$ & $0,77 \mathrm{D}$ & $\mathrm{R}$ & BR & MR & MR \\
\hline Alfa 200 & $1,18 \mathrm{C}$ & $0,81 \mathrm{D}$ & $\mathrm{R}$ & MR & MR & $\mathrm{R}$ \\
\hline WL 516 & $1,18 \mathrm{C}$ & $0,94 \mathrm{~B}$ & $\mathrm{R}$ & $\mathrm{R}$ & $\mathrm{R}$ & $\mathrm{R}$ \\
\hline El Grande & $1,13 \mathrm{C}$ & $1,08 \mathrm{~A}$ & $\mathrm{R}$ & MR & MR & $\mathrm{R}$ \\
\hline P 5888 & $1,12 \mathrm{C}$ & $0,81 \mathrm{D}$ & BR & $\mathrm{S}$ & MR & $\mathrm{R}$ \\
\hline BR2 & $1,12 \mathrm{C}$ & $0,94 \mathrm{~B}$ & MR & MR & MR & MR \\
\hline P 30 & $1,11 \mathrm{C}$ & $1,06 \mathrm{~A}$ & $\mathrm{R}$ & $\mathrm{R}$ & $\mathrm{R}$ & $\mathrm{R}$ \\
\hline MH4 & $1,10 \mathrm{C}$ & $0,75 \mathrm{D}$ & $\mathrm{BR}$ & $\mathrm{BR}$ & MR & MR \\
\hline P 5929 & $1,09 \mathrm{C}$ & $0,93 \mathrm{~B}$ & $\mathrm{R}$ & $\mathrm{BR}$ & MR & MR \\
\hline P 5715 & $1,01 \mathrm{C}$ & $0,85 \mathrm{C}$ & $\mathrm{R}$ & MR & MR & MR \\
\hline
\end{tabular}

(1)Médias seguidas pela mesma letra na coluna não diferem entre si a $5 \%$ de probabilidade pelo teste de Scott-Knott; R: Resistente; MR: Moderadamente Resistente; BR: Baixa resistência; S: Susceptível. 
na qualidade da forragem (Tabelas 1 e 2). Considerando, em conjunto, as duas estações do ano, verifica-se que somente as cultivares Crioula, WL 516 e P 30 foram consideradas resistentes à antracnose (Colletotrichum triffolii), cercosporiose (Cercospora medicaginis) e ferrugem (Uromices striatus), que foram as principais doenças observadas.

As cultivares foram consideradas resistentes a moderadamente resistentes ao pulgão-verde (Acyrthosiphon pisum), que foi a praga mais freqüente, principalmente durante o período da seca. Assim, durante a estação das águas não se observou a incidência do pulgão-verde em $70 \%$ das cultivares, e, no período da seca, somente $23 \%$ foram consideradas resistentes. Resultados semelhantes foram obtidos por Viana et al. (1998) na região de Cerrados, onde a ocorrência do pulgão-verde foi mais freqüente no período de inverno.

\section{Conclusões}

1. Sob condições de irrigação por aspersão, a alfafa apresenta boa oferta de forragem de alto valor nutritivo, ao longo do ano.

2. As cultivares Crioula, P30 e Monarca são recomendadas para o cultivo na região da Zona da Mata de Minas Gerais.

\section{Referências}

ALVIM, M. J.; BOTREL, M. A.; NOVELLY, P. E. Produção de gramíneas tropicais e temperadas, irrigadas na época da seca. Revista da Sociedade Brasileira de Zootecnia, Viçosa, MG, v. 5, p. 384-392, 1986

ASSOCIATION OF OFFICIAL ANALYTICAL CHEMISTS (Gaithersburg, Estados Unidos). Official methods of analysis. 11. ed. Washington, 1970. 1015 p.

BOLLAND, E. J. Utilización de alfalfa en producción de leche. In: WORKSHOP SOBRE O POTENCIAL FORRAGEIRO DE ALFAFA (Medicago sativa L.) NOS TRÓPICOS, 1994, Juiz de Fora. Anais... Juiz de Fora: Embrapa-CNPGL, 1994. p. 201-203.

BOTREL, M. A.; ALVIM, M. J. Avaliação de cultivares de alfafa na Zona da Mata de Minas Gerais. Pesquisa Agropecuária Brasileira, Brasília, v. 32, n. 9, p. 971-975, set. 1997
BOTREL, M. A.; ALVIM, M. J. Freqüência de corte da alfafa (cv. Crioula) na Zona da Mata de Minas Gerais Revista da Sociedade Brasileira de Zootecnia, Viçosa, MG, v. 25, n. 3, p. 396-403, 1996.

BOTREL, M. A.; ALVIM, M. J.; XAVIER, D. F. Efeito da irrigação sobre algumas características agronômicas de acessos de capim-elefante. Pesquisa Agropecuária Brasileira, Brasília, v. 26, n. 10, p. 1731-1736, out. 1991.

CASTILLO, R. A.; GALLARDO, R. M. Suplementación de vacas lecheras en pastoreo de alfalfa, concentrados y forrajes conservados. In: HIJANO, E. J.; NAVARRO, A. (Ed.). La alfalfa en la Argentina. Corrientes: Instituto Nacional de Tecnologia Agropecuária, 1995. p. 195-204.

COMERÓN, E. Sistemas de utilización de alfalfa para ganado lechero. In: WORKSHOP SOBRE O POTENCIAL FORRAGEIRO DE ALFAFA (Medicago sativa L.) NOS TRÓPICOS, 1994, Juiz de Fora. Anais... Juiz de Fora: Embrapa-CNPGL, 1994. p. 195-199.

EUCLYDES, R. F. Sistemas para análises estatísticas e genéticas (SAEG). Viçosa, MG: UFV, 1988. 68 p.

EVANGELISTA, A. R.; SALES, E. C. S.; CONÇALVES, F. G.; MOULIN, A. F.; JUNQUEIRA, G. D. Produção de 34 cultivares de alfafa com dois anos de cultivo no sul de Minas Gerais. In: REUNIÃO ANUAL DA SOCIEDADE BRASILEIRA DE ZOOTECNIA, 35., 1998, Botucatu. Anais... Botucatu: Sociedade Brasileira de Zootecnia, 1998. 1 CD-ROM

HIJANO, E. H. Metodología de evaluación de cultivares de alfalfa. In: WORKSHOP SOBRE O POTENCIAL FORRAGEIRO DAALFAFA (Medicago sativa L.) NOS TRÓPICOS, 1994, Juiz de Fora. Anais... Juiz de Fora: Embrapa-CNPGL, 1994. p. 23-28.

MARTEN, G. C.; BUXTON, D. R.; BARNES, R. F. Feeding value (forage quality). In: HANSON, A. A.; BARNES, D. K.; HILL, R. R. Alfalfa and alfalfa improvement. Madison: American Society of Agronomy, 1988. p. $465-484$

MELTON, B.; MOUNTRAY, J. B.; BOUTON, J. H. Geographic adaptation and cultivar selection. In: HANSON, A. A.; BARNES, D. K.; HILL, R. R. Alfalfa and alfalfa improvement. Madison: American Society of Agronomy, 1988. p. 596-618

RASSINI, J. B.; FREITAS, A. R. Desenvolvimento da alfafa (Medicago sativa, L.) sob diferentes doses de adubação potássica. Revista Brasileira de Zootecnia, Viçosa, $M G$, v. 27, n. 3, p. 487-490, 1998.

Pesq. agropec. bras., Brasília, v. 36, n. 11, p. 1437-1442, nov. 2001 
RASSINI, J. B.; FREITAS, A. R. Efeitos da interferência de plantas daninhas no rendimento da cultura da alfafa (Medicago sativa, L). Revista Brasileira de Zootecnia, Viçosa, MG, v. 24, n. 4, p. 502-509, 1995.

RUGGIERI, A. C.; CARVALHO, D.; FIGUEIREDO, L.; SANTOS, J. N.; CYRILLO, G.; RODRIGUES, L. A.; MONTEIRO, A. L.; MALHEIROS, E. B. Avaliação de 35 cultivares de alfafa em três diferentes períodos. In: REUNIÃOANUAL DA SOCIEDADE BRASILEIRADE ZOOTECNIA, 35., 1998, Botucatu. Anais... Botucatu: Sociedade Brasileira de Zootecnia, 1998. 1 CD-ROM.

VIANA, M. C. M.; KONZEN, E.; PURCINIO, H. M. A. Comportamento de 28 cultivares de alfafa nas condições de Cerrado de Sete Lagoas. In: REUNIÃO ANUAL DA SOCIEDADE BRASILEIRA DE ZOOTECNIA, 35. 1998, Botucatu. Anais... Botucatu: Sociedade Brasileira de Zootecnia, 1998. 1 CD-ROM.

VILELA, D. Potencial do pasto de alfafa (Medicago sativa $\mathrm{L}$.) para produção de leite. In: WORKSHOP SOBRE O POTENCIAL FORRAGEIRO DE ALFAFA (Medicago sativa L.) NOS TRÓPICOS, 1994, Juiz de Fora. Anais... Juiz de Fora: Embrapa-CNPGL, 1994. p. 171-185.

ZOCCAL, R. Leite em números. Coronel Pacheco: Embrapa-CNPGL/FAEMG, 1994. 131 p. 